Draft VERSION June 11, 2021

Preprint typeset using $\mathrm{LAT}_{\mathrm{E} X} \mathrm{X}$ style emulateapj v. 5/2/11

\title{
ON DETECTING MILLISECOND PULSARS AT THE GALACTIC CENTER
}

\author{
JeAn-Pierre MaCquarT ${ }^{1,2}$ AND Nissim KaneKar ${ }^{3}$ \\ Draft version June 11, 2021
}

\begin{abstract}
The lack of detected pulsars at the Galactic Center (GC) region is a long-standing mystery. We argue that the high stellar density in the central parsec around the GC is likely to result in a pulsar population dominated by millisecond pulsars (MSPs), similar to the situation in globular cluster environments. Earlier GC pulsar searches have been largely insensitive to such an MSP population, accounting for the lack of pulsar detections. We estimate the best search frequency for such an MSP population with present and upcoming broad-band radio telescopes for two possible scattering scenarios, the "weak-scattering" case suggested by the recent detection of a magnetar close to the GC, and the "strong-scattering" case, with the scattering screen located close to the GC. The optimal search frequencies are $\approx 8 \mathrm{GHz}$ (weak-scattering) and $\approx 25 \mathrm{GHz}$ (strong-scattering), for pulsars with periods $1-20 \mathrm{~ms}$, assuming that GC pulsars have a luminosity distribution similar to that those in the rest of the Milky Way. We find that 10-30 hour integrations with the Very Large Array and the Green Bank Telescope would be sufficient to detect MSPs at the GC distance in the weak-scattering case. However, if the strong-scattering case is indeed applicable to the GC, observations with the full Square Kilometre Array would be needed to detect the putative MSP population.
\end{abstract}

Subject headings: Galaxy: centre - pulsars:

\section{INTRODUCTION}

The allure of testing General Relativity near a supermassive black hole and investigating the latter's accretion environment (see, e.g., Pfahl \& Loeb 2004; Liu et al. 2012) has provided the motivation for numerous searches for radio pulsars at the Galactic Center (GC) over the last two decades (e.g. Johnston et al. 2006; Deneva 2010; Macquart et al. 2010). Yet, despite predictions that $100-1000$ radio pulsars with orbital periods $\lesssim 100$ years are orbiting Sgr A* (Pfahl \& Loeb 2004; Wharton et al. 2012; Chennamangalam \& Lorimer 2014; Zhang et al. 2014), not a single normal pulsar has so far been detected within $10^{\prime}$ of Sgr A*.

A long-standing problem related to these pulsar surveys relates to the scattering environment at the GC. The extreme angular broadening exhibited by Sgr A* and $\mathrm{OH} / \mathrm{IR}$ stars in the GC region (e.g. Lo et al. 1985; Frail et al. 1994), coupled with inferences about the distribution of the scattering material, led to the conclusion that the pulse smearing caused by turbulence along the line of sight to the GC is extreme, with a temporal smearing time of $\approx 10^{3} \nu_{\mathrm{CHz}}^{-4} \mathrm{~s}$, where $\nu_{\mathrm{GHz}}$ is the observing frequency in GHz (Cordes \& Lazio 1997; Lazio \& Cordes 1998). Such a large temporal smearing time would obstruct the detection of pulsed radio emission at the usual low frequencies at which pulsar searches are usually carried out (due to their steep radio spectrum). Indeed, for normal pulsars, with periods $\approx 0.5 \mathrm{~s}$, the temporal smearing would become negligible only at frequencies above $15 \mathrm{GHz}$ (Macquart et al. 2010), where the steep-

\footnotetext{
${ }^{1}$ ICRAR/Curtin University, Curtin Institute of Radio Astronomy, Perth WA 6845, Australia J.Macquart@curtin.edu.au ${ }^{2}$ ARC Centre of Excellence for All-Sky Astrophysics (CAASTRO)

${ }^{3}$ Swarnajayanti Fellow, National Centre for Radio Astrophysics, Tata Institute of Fundamental Research, Ganeshkhind, Pune - 411007, India
}

ness of the pulsar emission renders them weak and hence difficult to detect, even with today's most sensitive telescopes.

The recent detection of the GC magnetar PSR J174529 , located within $3^{\prime \prime}$ of the position of $\mathrm{Sgr} \mathrm{A} *$ (Kennea et al. 2013; Mori et al. 2013), has caused an upheaval in the field, lending renewed impetus to searches for pulsars close to $\operatorname{Sgr} A^{*}$. Spitler et al. (2014) reported the detection of pulsed emission from the magnetar at frequencies as low as $1.1 \mathrm{GHz}$, inferring a temporal smearing timescale of only $(1.3 \pm 0.2) \nu_{\mathrm{GHz}}^{-4} \mathrm{~s}$. The effect of pulse smearing on at least one object in the GC environment is thus three orders of magnitude smaller than that inferred from earlier studies. The magnetar also exhibits the same degree of angular broadening as Sgr A* (Bower et al. 2014), suggesting that it lies behind the same hyperturbulent scattering region. This hypothesis is supported by the high rotation measure of the magnetar (Eatough et al. 2013; Shannon \& Johnston 2013), which indicates that its proximity to $\mathrm{Sgr} \mathrm{A}^{*}$ on the sky is not merely a chance alignment.

The detection of the magnetar close to the GC suggests that the pulse smearing towards the $\mathrm{GC}$ is also relatively benign, assuming that the scattering screen is uniform. This would imply that radiation from ordinary pulsars at the GC should be readily detectable at frequencies $\gtrsim 3 \mathrm{GHz}$. However, if the effects of pulse smearing are similarly small over the entire GC region, previous surveys should have detected a significant fraction of the pulsar distribution with "normal" spin periods (Wharton et al. 2012; Dexter \& O'Leary 2014). This dearth of pulsar detections is particularly acute given that magnetars are believed to represent only $\sim 0.2 \%$ of all radio pulsars (Olausen \& Kaspi 2014; Chennamangalam \& Lorimer 2014). The lack of "slow" pulsar detections suggests that they either constitute an unusually small fraction of the entire GC pulsar popula- 
tion, or are significantly under-luminous relative to the population of slow pulsars that have been detected elsewhere in the Galaxy.

In passing, we note that Chennamangalam \& Lorimer (2014) used a Bayesian analysis combined with an assumed log-normal pulsar luminosity function to find that existing pulsar surveys at the GC are not sufficiently deep to eliminate the possibility that a substantial population of non-recycled low-luminosity pulsars might exist at the GC. While this is broadly correct, the shape of the pulsar luminosity function is not at all well-constrained at the low-luminosity end. As such, there is considerable error in the extrapolation of the distribution to low luminosities, and hence on such estimates of the total size of the normal pulsar population in the central parsec. For example, an uncertainly of only $20 \%$ in the two parameters of the log-normal distribution Bagchi et al. 2011; Chennamangalam \& Lorimer 2014) yields an uncertainty of two orders of magnitude in the survey completeness. Similar estimates of the number of potential MSPs at the GC are plagued with an even greater degree of uncertainty because the luminosity function of recycled pulsars in globular clusters is even less well known. In the present analysis, we will adopt a pragmatic approach to the pulsar luminosity distribution of pulsars: our detection arguments are made only with reference to the known luminosities of detected Galactic pulsars.

In this paper, we explore the reasons for the paucity of pulsar detections at the Galactic Center. In $\S 2$, we discuss the nature of the pulsar population at the GC region, and argue that it is likely to be dominated by recycled millisecond pulsars (MSPs); present pulsar surveys of the GC have been largely insensitive to such a population. Next, we present in $\$ 3$ detailed calculations of the expected signal-to-noise ratio for a wide-bandwidth search for MSPs at the Galactic Center with present and future telescopes, incorporating the frequency dependence of temporal smearing and sky temperature across the different observing bands. Finally, the results of this work are summarized in $\S 4$.

\section{A MILLISECOND PULSAR POPULATION AT THE GALACTIC CENTER}

Over the last two decades, several searches have been carried out for pulsars at the GC, mostly at frequencies between $1.4 \mathrm{GHz}$ and $8 \mathrm{GHz}$ (e.g. Johnston et al. 1995, 2006; Kramer et al. 2000; Deneva et al. 2009; Deneva 2010; Bates et al. 2011). Based on the high expected temporal smearing, Macquart et al. (2010) argued that higher observing frequencies are more amenable to the discovery of pulsars, with the optimal frequency range for searches for "normal" pulsars - those with periods of $\approx 0.5$ seconds - being $10-16 \mathrm{GHz}$. Following this, there have been a number of deep searches at frequencies above $10 \mathrm{GHz}$, at $15 \mathrm{GHz}$ by Macquart et al. (2010), at $12-18 \mathrm{GHz}$ by Siemion et al. (2013), and at $19 \mathrm{GHz}$ by Eatough et al. (2013). Remarkably, despite integration times exceeding 10 hours with $100-\mathrm{m}$ single-dish telescopes, none of these searches has discovered a single pulsar in the Galactic Centre region!

Using the temporal smearing estimates of Lazio \& Cordes (1998), Macquart et al. (2010) estimated that their $15 \mathrm{GHz}$ search would have been sensitive to $\approx 15 \%$ of the Galactic center pulsar

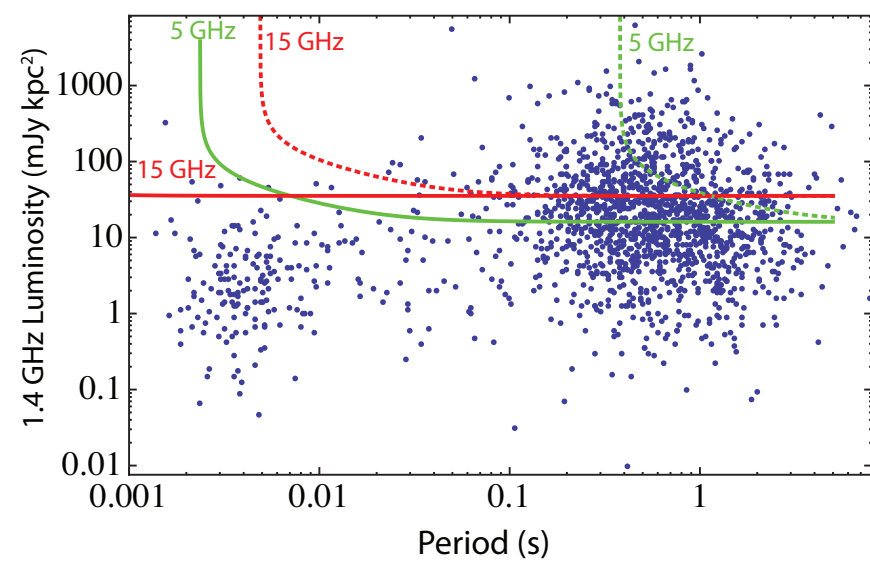

FIG. 1.- The $1.4 \mathrm{GHz}$ luminosity (in $\mathrm{mJy} \mathrm{kpc}^{2}$ ) of the known pulsar population (Manchester et al.2005) is plotted versus pulsar period. The $10 \sigma$ sensitivities of previous $5 \mathrm{GHz}$ (Johnston et al. 2006) and $15 \mathrm{GHz}$ GBT (Macquart et al. 2010) searches of the Galactic Center are shown by the green and red curves, respectively, with the dashed and solid curves representing the "strong" and "weak" temporal smearing scenarios, respectively.

population, assuming the luminosity distribution of known Galactic pulsars. However, if the pulse smearing is benign, as suggested by the detection of the GC magnetar, then even the earlier lower-frequency searches would have been sensitive to normal pulsars located near the GC. Indeed, Dexter \& O'Leary (2014) estimate that both the $5 \mathrm{GHz}$ search of Johnston et al. (2006) and the $15 \mathrm{GHz}$ search of Macquart et al. (2010) would both have been sensitive to $\approx 20 \%$ of the GC pulsar population, if its characteristics resemble those of the known pulsar population (see Fig. 2 of Dexter \& O'Leary 2014). This "missing pulsar" problem was used by Dexter \& O'Leary (2014) to argue that the GC pulsar population may be dominated by magnetars, i.e. that the population is very different from that in the rest of the Galaxy (where, as noted earlier, only $\approx 0.2 \%$ of known radio pulsars are magnetars, although the magnetar birth rate may be $10-50 \%$ of the total neutron star birth rate in the Galaxy; e.g. Keane \& Kramer 2008).

However, a number of studies have raised another possibility, namely that the GC pulsar population is dominated by recycled millisecond pulsars (MSPs). The dense stellar environment at the GC is likely to result in spinning pulsars up to millisecond periods by frequent close interactions with neighbouring stars, by analogy with the population of MSPs detected in the dense stellar environments of globular clusters (e.g. Alpar et al. 1982; Verbunt 1987; Camilo et al. 2000; Ransom et al. 2005). Note that the stellar density in the central parsec of the $\mathrm{GC}$ is $\approx 10^{6}$ per cubic parsec (e.g. Genzel et al. 1996; Schödel et al. 2007), a couple of orders of magnitude larger than the stellar density in globular cluster cores $\left(\lesssim 10^{4}\right.$ per cubic parsec), implying that close interactions are far more likely in the vicinity of the GC. The formation rate of low-mass X-ray binaries (LMXBs) in dense environments is also proportional to both the number density of neutron stars and the stellar density (Verbunt \& Hut 1987). Since the neutron star density itself scales with the stellar density, this implies that the formation rate of LMXBs (and hence, that of 
binary pulsars; e.g. Alpar et al. 1982; Campana et al. 1998) is roughly proportional to the square of the stellar density. When coupled with the higher stellar density at the GC, this implies a far higher formation rate of LMXBs (and binary pulsars) than in globular cluster cores. We note that more than $90 \%$ of all known globular cluster pulsars have periods smaller than $30 \mathrm{~ms}$ (Manchester et al. 2005), indicating that a dense environment can dramatically alter the period distribution. Further, an over-abundance of X-ray transients has been detected within 1 pc of Sgr A* (Muno et al. 2005); these appear to be LMXBs produced by three-body interactions between stellar binaries and either black holes or neutron stars located in the central parsec (similar to the case in globular cluster cores; Muno et al. 2005). Indeed, Faucher-Giguère \& Loeb (2011) argue that the GC environment is likely to produce pulsar-black hole binary systems, via three-body interactions between stellar black holes and recycled pulsar binaries; the resulting pulsars would have periods in the MSP range. Note that, as emphasized by Faucher-Giguère \& Loeb (2011), their estimate of ten times more MSPs in the GC region than in Terzan 5 is likely to only be good to within an order of magnitude. Overall, though, it appears quite plausible that the GC pulsar population is dominated by recycled MSPs.

A possible caveat to the above argument is that globular clusters are not undergoing active star formation. As a result, neutron stars in globular clusters are "old" systems; non-recycled pulsars would have long-since spun down, so the only remaining pulsars are likely to be recycled MSPs. This is an important factor in the dominance of MSPs in the globular cluster population. In the case of the GC, there is certainly active star formation in the vicinity of $\operatorname{Sgr} \mathrm{A}^{*}$, and hence there may well be a sizeable population of young pulsars. However, the population of neutron stars left over from the many earlier generations of star formation at the GC is likely to outnumber the population from the current generation of star formation. This earlier population is likely to have been spun up, and would now contribute to the present MSP population. In addition, the much higher stellar density at the GC than in globular clusters (by about two orders of magnitude) implies that the scattering timescales are far shorter in the GC environment. Thus, even if there is a population of young pulsars in the GC environment, they would be spun up to MSPs much more quickly than in globular clusters; the result is that MSPs should still dominate the GC pulsar population.

Fig. 1 overlays the sensitivity of the best present searches (Johnston et al. 2006; Macquart et al. 2010) on the luminosity-period distribution of the known pulsar population, assuming the "weak scattering" scenario, i.e. that GC pulsars are subject to the same temporal smearing as the GC magnetar. While the $5 \mathrm{GHz}$ search of Johnston et al. (2006) is more sensitive to normal pulsars than the $15 \mathrm{GHz}$ search of Macquart et al. (2010), even the relatively-benign assumed temporal smearing causes a significant reduction in its sensitivity to fast pulsars with periods $<10 \mathrm{~ms}$. Further, even the $15 \mathrm{GHz}$ search of Macquart et al. (2010) is sensitive to only a small fraction $(\lesssim 4 \%)$ of a GC MSP population whose properties resemble those of known MSPs. We emphasize that this assumes the optimistic scenario in which the temporal smearing towards GC pulsars is similar to that measured towards the GC magnetar. The dashed curves in Fig. 1 show that the situation is even worse if the GC magnetar is being seen through a "hole" in the screen, and the temporal smearing towards $\mathrm{Sgr} \mathrm{A}^{*}$ is similar to the earlier estimates: the $5 \mathrm{GHz}$ and $15 \mathrm{GHz}$ searches would then be entirely insensitive to pulsars of periods $\lesssim 40 \mathrm{~ms}$. Thus, given that the GC pulsar population is likely to be dominated by MSPs and that present searches have been insensitive to such a population, it appears that an MSP population is a viable way of hiding pulsars at the GC, and solving the missing pulsar problem.

In passing, we note that the 2 brightest MSPs in Terzan 5 would have been detected in the $15 \mathrm{GHz}$ survey of Macquart et al. (2010), if located at the distance of the GC, and if the weak scattering scenario indeed applies to the GC pulsar population. The lack of detections in this survey appears surprising if the $\mathrm{GC}$ region indeed has $\approx 10$ times the number of MSPs seen in Terzan 5 (Faucher-Giguère \& Loeb 2011). Indeed, this might be considered evidence that either the GC sightline is subject to strong scattering or that the number of MSPs at the GC has been over-estimated. However, we caution that small number statistics makes it difficult to extrapolate from two bright MSPs in Terzan 5 to the full GC population. It is hence critical to increase the sensitivity of GC searches so as to be able to detect a significant fraction of the Terzan 5 (or known MSP) population, if placed at the GC distance. If MSPs remain undetected in such searches, the non-detections would essentially imply that either the weak scattering case is not applicable to the GC or that the GC pulsar population is somehow very different from that in globular cluster environments.

\section{THE DETECTABILITY OF PULSARS AT THE GALACTIC CENTER}

In this section, we compute in detail the expected signal-to-noise ratio for MSPs at the Galactic Center with the most sensitive current and forthcoming radio telescopes. These computations are motivated both by the arguments of the preceding section concerning MSPs at the Galactic Center and by the dramatic increase in the fractional bandwidth of modern pulsar backends (e.g. Siemion et al. 2013), due to which the frequency dependence of the relevant quantities (e.g. Lazio \& Cordes 1998; Macquart et al. 2010) within each observing band plays a critical role in determining the optimal observing frequency. The following analysis takes into account variations in the signal-to-noise ratio $(\mathrm{S} / \mathrm{N})$ across the observing band due to the pulsar radio spectrum, the frequency dependence of the system temperature, and the change in the pulse width at different frequencies caused by temporal smearing.

\subsection{The $S / N$ for wide bandwidth pulsar searches at the Galactic Center}

For a narrow observing bandwidth, $\Delta \nu$, the $\mathrm{S} / \mathrm{N}$ for the detection of a pulsar of average flux density $S_{\nu}$, pulse period $P$ and width $W$ is (Lorimer et al. 2006)

$$
S / N=S_{\nu} \frac{G \sqrt{n_{p} \Delta \nu \Delta t}}{T_{\mathrm{sys}}} \sqrt{\frac{P-W}{W}},
$$


where $\Delta t$ is the telescope integration time, $n_{p}$ is the number of polarizations observed, $G$ is the telescope gain and $T_{\text {sys }}$ is the total system temperature. Note that the above equation assumes that $S_{\nu}, T_{\text {sys }}$ and $W$ do not depend on the observing frequency. However, when the observing bandwidth is large, this assumption breaks down and Equation (1) must be generalised to incorporate the frequency dependence of both the noise and the signal across the observing band. The resulting net $\mathrm{S} / \mathrm{N}$ can be estimated by comparing the total signal in the observing band to the total noise received during the duration of the observations. The total signal measured over the frequency interval $\left(\nu_{1}, \nu_{2}\right)$ in a time period $\Delta t$ is then

$$
S=n_{p} \Delta t \int_{\nu_{1}}^{\nu_{2}} S_{\nu}\left(\nu^{\prime}\right) d \nu^{\prime}
$$

Next, the noise power per polarization in a single time and frequency channel of respective widths $\delta t$ and $\delta \nu$ is $n=T_{\text {sys }}(\nu) \sqrt{\delta t \delta \nu} / G$. For observations over a number $N_{\text {ch }}$ of such time, frequency and polarization channels, the noise adds in quadrature to give

$$
N=\sqrt{\sum_{i}^{N_{\mathrm{ch}}} n_{i}^{2}}
$$

For an observation with $n_{p}$ polarizations, covering a frequency interval $\left(\nu_{1}, \nu_{2}\right)$, and of duration $\Delta t$, the above can be generalized to

$$
N=\sqrt{\Delta t n_{p} \int_{\nu_{1}}^{\nu_{2}} \frac{T_{\mathrm{sys}}^{2}\left(\nu^{\prime}\right)}{G^{2}\left(\nu^{\prime}\right)}\left[\frac{W\left(\nu^{\prime}\right)}{P-W\left(\nu^{\prime}\right)}\right] d \nu^{\prime}} .
$$

In the above equation, the factor $W /(P-W)$ inside the integral represents the fraction of power present in the pulsed signal per unit time.

Next, the telescope system temperature $T_{\text {sys }}$ contains contributions from the sky and the telescope receiver temperature $T_{\text {rec }}$, each of which has different dependences on frequency. The sky temperature itself contains two contributions, one from the bright GC region, which we label $T_{\mathrm{GC}}$, and the other from the atmosphere, $T_{\mathrm{atm}}$. The atmospheric contribution depends on the telescope site and increases with frequency at frequencies $\gtrsim 20 \mathrm{GHz}$. It is convenient to absorb the two telescopedependent contributions, from the telescope electronics and the atmosphere above the site, into a single term which we label $T_{\mathrm{R}}$, so that the total system temperature is expressed in the form

$$
T_{\text {sys }}(\nu)=T_{\mathrm{GC}}(\nu)+T_{\mathrm{R}}(\nu)
$$

The determination of the GC contribution to the sky temperature is complicated by the fact that the emission near Sgr $A^{*}$ has a strong spatial dependence, so that the exact amplitude and spectrum of the contribution depends on the telescope beam size (which itself depends on frequency). For simplicity, we model the decline of sky temperature with angular distance $\theta$ from Sgr A* using a Gaussian profile,

$$
T(\theta, \nu)=T_{0}(\nu) \exp \left[-\frac{\theta^{2}}{\theta_{0}^{2}}\right],
$$

where $T_{0}(\nu)=350(\nu / 2.7 \mathrm{GHz})^{-2.7} \mathrm{~K}$ (Reich et al. 1990). We further estimate $\theta_{0}=0.33^{\circ}$ from the $2.7 \mathrm{GHz}$ image of Reich et al. (1990). We emphasize that this approximation only applies in the neighbourhood of Sgr A* but is sufficient for our purposes, given the high frequencies ( $\gtrsim 5 \mathrm{GHz}$ ), and hence, the relatively small telescope beamwidths under consideration here. The effective contribution of the sky temperature to the system temperature is determined by computing the weighted average of the sky emission over the telescope beam, $B(\boldsymbol{\theta})$ :

$$
T_{\mathrm{GC}}(\nu)=\frac{\int T(\boldsymbol{\theta}) B(\boldsymbol{\theta}) d^{2} \boldsymbol{\theta}}{\int B(\boldsymbol{\theta}) d^{2} \boldsymbol{\theta}}=T_{0}(\nu)\left(\frac{\theta_{0}^{2}}{\theta_{0}^{2}+\theta_{b}^{2}}\right),
$$

where, in the second equality, we assume a gaussian beam shape of full-width-at-half-maximum $\theta_{b}=1.22 \lambda / d$, where $d$ is the telescope diameter or, in the case of an interferometer, the diameter of each element of the array.

We have computed the $\mathrm{S} / \mathrm{N}$ values for the two most sensitive present telescopes covering the frequency range $\approx 5-50 \mathrm{GHz}$, the Green Bank Telescope (GBT) and the Karl G. Jansky Very Large Array (VLA). For these telescopes, the system temperature (not including the contribution from the GC region) at the low GC elevations, the telescope gain and the usable bandwidth in different observing bands have been obtained from the telescope webpages. In addition, we also consider the proposed sensitivities of the mid-frequency array of the Square Kilometre Array Phase-1 (SKA1-MID) and the full Square Kilometre Array (SKA) (Dewdney et al. 2013).

We consider two scattering scenarios: "weak scattering", where the pulse smearing timescale is assumed to be the same as that measured towards the GC magnetar (Spitler et al. 2014)

$$
\tau_{\text {smear }}=1.3 \times\left(\frac{\nu}{1 \mathrm{GHz}}\right)^{-4} \mathrm{~s},
$$

and "strong scattering", where the scattering occurs $130 \mathrm{pc}$ from the GC (Lazio \& Cordes 1998) and, using the GC distance and angular broadening size of Sgr $\mathrm{A}^{*}$ adopted by Bower et al. (2014), the pulse smearing timescale is given by

$$
\tau_{\text {smear }} \approx 2.1 \times 10^{2} \times\left(\frac{\nu}{1 \mathrm{GHz}}\right)^{-4} \mathrm{~s} .
$$

The observed pulse width is the sum in quadrature of the pulse scattering timescale, the intrinsic pulse width and the detector channel width:

$$
W=\sqrt{\left(w_{50} P\right)^{2}+\tau_{\text {smear }}^{2}+\delta t^{2}},
$$

where the intrinsic pulse width is taken to be a fixed fraction, $w_{50}$, of the pulse period. The time resolution of the detector, $\delta t$, is an important factor when the detector time resolution is coarser than the intrinsic pulse width. In this regime, we see from Equation (10) that, in the limit of narrow pulse widths, the observed pulse width tends to the detector time resolution, $\delta t$, and that no further improvement in $\mathrm{S} / \mathrm{N}$ is possible with a further decrease in $w_{50} P$. We will assume a time resolution of $50 \mu \mathrm{s}$ for the VLA, SKA1-MID and full SKA, which is

\footnotetext{
4 https://science.nrao.edu/facilities/vla https://science.nrao.edu/facilities/gbt
}

and 
more than sufficient to not hinder the detection of MSPs. However, in the case of the GBT, we have assumed a time resolution of $0.5 \mathrm{~ms}$, the best available with the present wideband VEGAS spectrometer; this does influence the detectability of the fastest MSPs, with periods of $\approx 1 \mathrm{~ms}$.

We note that Equation (1), and its generalization for large bandwidths, implies that a pulsar whose pulse width $W$ matches the pulse period $P$ would be completely undetectable, with the noise term increasing rapidly as $W$ approaches $P$. When including the effect of scatter broadening on the pulse width, Equation (10) implicitly assumes that the scatter-broadened pulse profile is smeared into a boxcar of width $W$, rather than being convolved with an exponential tail. The latter case would result in some pulsed emission being visible even with $W>P$ (e.g. Macquart et al. 2010). A more accurate treatment would slightly improve the detectability of pulsars at lower frequencies, but would not significantly alter our results.

\subsection{Application to the Galactic Center}

Incorporating all the above considerations, the signalto-noise ratio for a pulsar at the Galactic Center is

$$
\mathrm{S} / \mathrm{N}=\frac{1.49 \times 10^{-5} \sqrt{n_{p} \Delta t} \int_{\nu_{1}}^{\nu_{2}} L_{1.4}\left(\frac{\nu}{1.4 \mathrm{GHz}}\right)^{-\alpha} d \nu^{\prime}}{\sqrt{\int_{\nu_{1}}^{\nu_{2}} \frac{T_{\mathrm{sys}}^{2}\left(\nu^{\prime}\right)}{G^{2}\left(\nu^{\prime}\right)}\left[\frac{W\left(\nu^{\prime}\right)}{P-W\left(\nu^{\prime}\right)}\right] d \nu^{\prime}}}(11)
$$

where we have defined the $1.4 \mathrm{GHz}$ pulsar (pseudo)luminosity $L_{1.4}=S_{\nu} d_{\mathrm{GC}}^{2}$ in units of $\mathrm{mJy} \mathrm{kpc}^{2}$, the numerical prefactor assumes a GC distance of $d_{\mathrm{GC}}=$ $8.2 \mathrm{kpc}$, and $\alpha$ is the pulsar spectral index $\left(S_{\nu} \propto \nu^{-\alpha}\right)$.

Analyses of MSP spectra indicate a mean spectral index in the range 1.6-1.8 Kramer et al. 1998; Maron et al. 2000); we hence adopt a typical value of $\alpha=1.7$ in our modelling. However, we note that a recent analysis of the slow pulsar population favours a lower spectral index, $\alpha=1.4$ (Bates et al. 2013). If this also applies to the MSP population, it would assist the detectability of pulsars at high frequencies.

The optimal detection frequency for a pulsar of a given period is chiefly determined by the competition between scattering, which broadens the pulses to a timescale less than the pulse period only above some frequency $\nu_{\text {lim }}$, and the pulsar spectrum, which declines with increasing frequency (of course, secondary considerations include the sky contribution to the system temperature, the telescope characteristics and the observing bandwidth). It is obvious that the pulsar is undetectable at frequencies below which the pulse broadening timescale matches the pulsar period. It might therefore be supposed that the optimal detection frequency includes frequencies just above $\nu_{\text {lim }}$. However, the noise contribution becomes arbitrarily large for frequencies in the vicinity of $\nu_{\text {lim }}$, where $P \approx W$. In fact, there is a range of frequencies above $\nu_{\text {lim }}$ where $W(\nu)$ is not sufficiently small compared to $P$, and where the contribution of the noise power per frequency interval in Equation (11) exceeds the contribution from the pulsed signal power, so that inclusion of this region decreases the $\mathrm{S} / \mathrm{N}$. It is therefore advantageous to restrict the lower bound of the observing band to exclude this region, so as to maximize the $\mathrm{S} / \mathrm{N}$. In our calculations of the $\mathrm{S} / \mathrm{N}$ for bands near $\nu_{\text {lim }}$, we therefore optimize the $\mathrm{S} / \mathrm{N}$ by restricting the lower cutoff frequency to include only those frequencies which make a positive contribution to the $\mathrm{S} / \mathrm{N}$ (i.e. we do not necessarily use the entire available bandwidth in the detection). Observationally, one would similarly attempt to maximize the $\mathrm{S} / \mathrm{N}$ of any detection by restricting the range of frequencies only to those in which pulsed power is evident.

The four panels of Figure 2 illustrate the detectability of MSPs of three different periods, $1 \mathrm{~ms}, 5 \mathrm{~ms}$ and $20 \mathrm{~ms}$ (all with $w_{50}=10 \%$ and $L_{1.4}=10 \mathrm{mJy} \mathrm{kpc}^{2}$, and located at the Galactic Center distance), with a 30hour integration with the four telescopes under consideration. The filled squares and solid curves correspond to the low-scattering magnetar-like scenario (with $\tau_{\text {smear }}=$ $\left.1.3 \nu_{\mathrm{GHz}}^{-4} \mathrm{~s}\right)$, while the open circles and dotted curves correspond to the high-scattering scenario arising from a screen located $130 \mathrm{pc}$ from $\operatorname{Sgr} \mathrm{A}^{*}\left(\tau_{\text {smear }}=208 \nu_{\mathrm{GHz}}^{-4} \mathrm{~s}\right)$.

It is clear from the figure that, in the weak-scattering case, the peak $\mathrm{S} / \mathrm{N}$ is obtained at a central frequency of $\approx 10 \mathrm{GHz}$ (i.e. the X-band for the GBT and the VLA) for MSPs with periods $\lesssim 10 \mathrm{~ms}$, while for slower $(20 \mathrm{~ms})$ pulsars, the peak $\mathrm{S} / \mathrm{N}$ shifts to $\approx 6 \mathrm{GHz}$ (i.e. the $\mathrm{C}$-band of the GBT and the VLA), and to progressively lower frequencies for progressively slower rotators. Conversely, in the high-scattering case, the peak $\mathrm{S} / \mathrm{N}$ for MSPs is obtained at much higher frequencies, $\approx 20-30 \mathrm{GHz}$ for MSPs with periods in the range $1-20 \mathrm{~ms}$.

In passing, we note that the large data volumes imply that a high statistical significance (typically, $\gtrsim 10 \sigma$ ) is usually required in such pulsar surveys. This implies that an MSP with $L_{1.4}=10 \mathrm{mJy} \mathrm{kpc}^{2}$ and with periods $<20 \mathrm{~ms}$ would be detectable with integration times of $\approx 10-30$ hours with the GBT and the VLA (and, of course, with the SKA1-MID and the full SKA) in the weak-scattering case. However, much larger integration times $(>100$ hours) would be needed on the GBT, the VLA or the SKA1-MID to detect such an MSP in the high-scattering case. If the GC pulsar population is indeed dominated by MSPs and the high-scattering case applies, it may only be possible to detect this population with the full SKA.

Of course, the optimal detection frequency for MSPs critically depends on both the MSP period distribution and the MSP luminosity function. Assuming that these are same for the GC environment as those of the known MSP population (e.g. Manchester et al. 2005), we can determine the overall optimal detection frequency for MSPs (integrated over all spin periods), by estimating, for each spin period, the frequency at which the peak $\mathrm{S} / \mathrm{N}$ occurs, and then plotting this frequency against the peak $\mathrm{S} / \mathrm{N}$ weighted by the relative fraction of MSPs with this period. The results of this analysis for the GBT, VLA and SKA1-MID are shown in Figure 3, in both the weak- and strong-scattering regimes. We note that the period distribution of the known MSP population is dominated by spin periods between 1 and $3 \mathrm{~ms}$. In the weak-scattering regime, the $\mathrm{S} / \mathrm{N}$ for the three telescopes is either relatively flat over the frequency range $5-10 \mathrm{GHz}$ or peaks at $\approx 10 \mathrm{GHz}$. It is hence not surprising that the optimal detection frequency in the weak-scattering regime closely matches that at which the $\mathrm{S} / \mathrm{N}$ is maximal for pulsars of those periods (i.e. $\approx 8-10 \mathrm{GHz}$ ). However, we note 

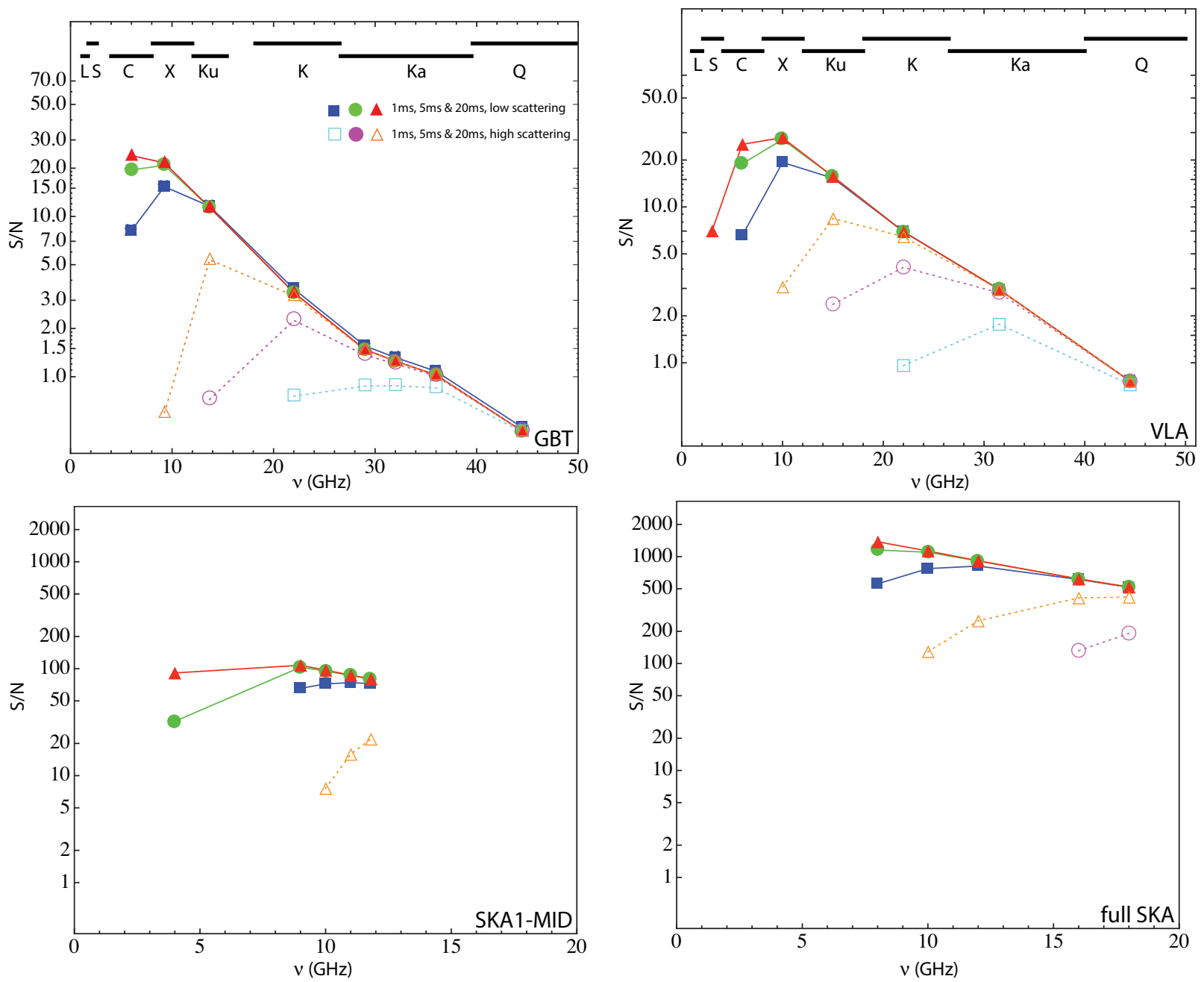

FIG. 2.- The frequency dependence of the S/N for GBT, VLA, SKA-MID and full SKA searches for MSPs at the Galactic Center. The filled and open symbols are, respectively, for the weak-scattering (i.e. magnetar-like) and strong-scattering scenarios. The symbols plotted are for MSPs with the listed periods (1,5, and $20 \mathrm{~ms}$ ), a duty cycle of $10 \%$ and a spectral index of -1.7 . The vertical axis gives the expected $\mathrm{S} / \mathrm{N}$ after a 30-hour integration for an MSP with a $1.4 \mathrm{GHz}$ luminosity of $L_{1.4}=10 \mathrm{mJy} \mathrm{kpc}^{2}$. The frequency ranges of the current suite of available receivers for each telescope is shown at the top of each panel. The optimal frequency band for MSPs with periods $\lesssim 10$ ms is $8-12 \mathrm{GHz}$ in the weak-scattering case, and $18-26 \mathrm{GHz}$ (i.e. K-band) in the strong-scattering case.

that in the strong-scattering regime, the optimal detection frequency is actually $\approx 15 \mathrm{GHz}$ for the VLA, i.e. lower than the frequency at which the $\mathrm{S} / \mathrm{N}$ is maximal for MSPs with periods of $1-3 \mathrm{~ms}$. This is because the VLA detection $\mathrm{S} / \mathrm{N}$ in the strong-scattering regime is far higher for MSPs with periods of $\approx 20 \mathrm{~ms}$ than for MSPs with periods of $\approx 1-3 \mathrm{~ms}$, and this compensates for the larger fraction of fast MSPs in the population.

We emphasize that the above analysis makes the critical assumption that both the MSP luminosity function and the MSP period distribution in the GC environment are the same as those of the known MSP population, dominated by MSPs in globular clusters. It is not implausible that these are different in the GC and globular cluster environments. Interactions that form MSPs in the GC region are likely to differ substantially from those that operate in other known environments (e.g. due to the increased prevalence of binary interactions in the GC region; Faucher-Giguère \& Loeb 2011).

Recognising that X-band is the optimal detection band for most GC MSPs in the weak-scattering regime, Fig- ure 4 shows the limiting detection luminosity, scaled to $1.4 \mathrm{GHz}$, for a 30 -hour survey of the Galactic Center as a function of pulse period. The $1.4 \mathrm{GHz}$ luminosities of known pulsars (from the ATNF pulsar catalogue; Manchester et al. 2005) are plotted for comparison. As noted earlier, previous pulsar surveys of the GC have been insensitive to all but the most luminous members of the known MSP population, if located at the GC distance: the most sensitive previous search (Macquart et al. 2010) would have detected only $4 \%$ of all known pulsars with periods $\leq 20 \mathrm{~ms}$. By contrast, a $30 \mathrm{~h}$ X-band survey with the GBT would detect $27 \%$, and a corresponding VLA survey $42 \%$, of this population. Of course, both such surveys would, in addition, be sensitive to $>80 \%$ of slow pulsars, with periods $\geq 0.1 \mathrm{~s}$.

\subsection{Searches for accelerated pulsars}

Implicit in the foregoing calculations and time estimates is the assumption that it is possible to integrate coherently for several tens of hours on individual pulsars. This is potentially an issue for MSPs, which tend 


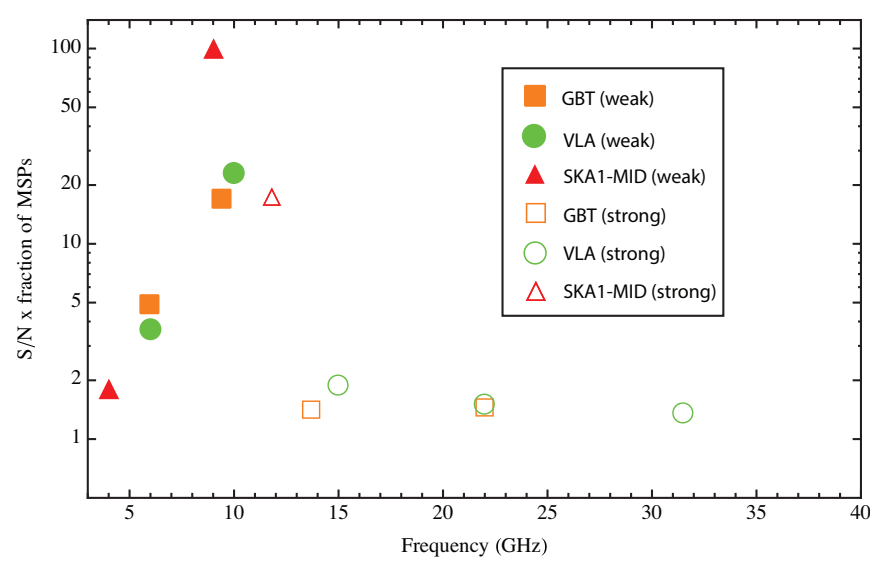

FIG. 3.- An estimate of the optimal detection frequency for MSPs with spin periods up to $50 \mathrm{~ms}$, under the assumption that the MSP period distribution at the GC is the same as that of the known MSP population. For each frequency band, the plot shows the fraction of pulsars with spin periods whose peak $\mathrm{S} / \mathrm{N}$ falls in this band, weighted by their peak detection $\mathrm{S} / \mathrm{N}$. The filled symbols designate values for the weak scattering case, while open symbols indicate the corresponding values for the strong scattering case.

to occur in binary systems and where the inherent orbital accelerations can lead to a non-negligible pulse phase drift for observations lasting longer than a small fraction of the orbital period. For example, more than half of the known MSPs (with periods $\leq 20 \mathrm{~ms}$ ) in globular clusters arise in binaries 5 , with orbital periods ranging from $\approx 1.6$ hours to $\approx 191$ days (e.g. Camilo et al. 2000; Ransom et al. 2005), and a median orbital period of $\approx 17$ hours. Searches for MSPs at the Galactic Center that extend for more than a few hours are hence likely to be affected by the above issue, which is relevant to about half the MSP population (assuming that the distribution between "isolated" and "binary" MSPs is the same as that in globular clusters). Pulse phase drift is a particularly important consideration for GC searches with the GBT and VLA, whose northern latitudes restrict them to individual GC observing runs of durations $\lesssim 6$ hours. A 30 -hour GBT or VLA integration on the GC would thus require that one integrate coherently over a period of at least 5 days.

Drifts in the pulse period over the duration of the observation must be taken into account, so that the sensitivity is not reduced for MSPs in short-period binaries. When determining the optimal method for taking the pulsar orbit into account in the detection process, it is convenient to characterise binary pulsars in terms of the ratio of total observation duration, $\Delta T$, to the orbital period, $T_{\text {orb }}$. For pulsars with $\Delta T / T_{\text {orb }}<0.1$, the change in pulse frequency is sufficiently small that standard acceleration searches are efficient (Wood et al. 1991; Ransom et al. 2002). Conversely, if $\Delta T / T_{\text {orb }} \gtrsim$ 1.5 , phase modulation searches may be employed at the penalty of a slight decrease in sensitivity (Jouteux et al. 2002; Ransom et al. 2003). However, in the intermediate regime $0.1 \lesssim \Delta T / T_{\text {orb }} \lesssim 1.5$, full sensitivity searches require a search over the Keplerian parameters of the orbit. For orbital periods similar to those typical of binary MSPs in globular clusters, searches over the orbital parameters would be needed for integrations lasting longer

\footnotetext{
${ }^{5}$ See http://www.naic.edu/ pfreire/GCpsr.html
}

than $\approx 1$ hour.

In the case of SKA1-MID and the full SKA, the relatively high sensitivity implies that short searches (of $\lesssim 1$ hour duration), in conjunction with existing acceleration search software, would be capable of detecting significant fractions of the known MSP population $(\approx 25 \%$ for SKA1-MID and $\approx 90 \%$ for the full SKA) if located at the GC distance. However, for the GBT and the VLA, such short integrations would only be able to detect a small fraction (a few \%) of the population, as the limiting luminosity would be a factor of $\approx 5.5$ worse than that of the 30-hour integrations shown in Fig. 4. One might increase the net statistical significance of detections by performing multiple searches over multiple short stretches of data. In such an approach, one would retain all candidates detected at a lower significance (i.e. $<10 \sigma$ ) and then jointly analyse all such marginal candidates to test whether the different observing epochs yield pulse periods and accelerations consistent with a fitted orbit.

The alternative approach would be to fit different combinations of the six Keplerian orbital parameters to the phase-coherent pulse data. The most effective current search methods in this regime have been developed and successfully implemented to detect binary pulsars on circular orbits Knispel 2011; Knispel et al. 2013). This technique relies on convolution of the data with multiple orbital parameter combinations; its extreme computational expense has necessitated implementation on highly distributed machines (e.g. Einstein@Home). A potential disadvantage of this approach is the increase in the dimensionality of the search over a conventional acceleration search, requiring a commensurate increase in the $\mathrm{S} / \mathrm{N}$ of any detection in order to achieve an acceptable level of significance. However, this can be partially mitigated by the restriction of the search to a specific range of orbital periods and to small eccentricities, as has been done in previous searches.

\section{CONCLUSIONS}

The high stellar density in the central parsec of the Milky Way offers a solution to the long-standing mystery of the lack of pulsar detections at the Galactic Center. Analogous to the situation in globular clusters (where the densities are a few orders of magnitude lower than at the GC), the high density is likely to result in a GC pulsar population dominated by millisecond pulsars. Previous GC pulsar searches have been almost entirely insensitive to such an MSP population.

We estimate the optimal search frequency for an MSP population for two present and two future telescopes, the VLA, the GBT, the SKA1-Mid and the full SKA, assuming that the GC pulsar population has a luminosity distribution similar to that of field pulsars. We consider two scattering cases, weak-scattering, where the scattering screen is roughly midway between us and the GC, and strong-scattering, where the screen is $\approx 130 \mathrm{pc}$ from the GC. We find that the optimal MSP search frequencies are $\approx 8 \mathrm{GHz}$ and $\approx 25 \mathrm{GHz}$ in the weak-scattering and strong-scattering cases, respectively. Deep (10 - 30 hour) integrations with the VLA or the GBT should allow a detection of MSPs at the GC at $\gtrsim 10 \sigma$ significance if the weak-scattering case is indeed applicable, as suggested by the recent detection of a magnetar close to the GC. How- 


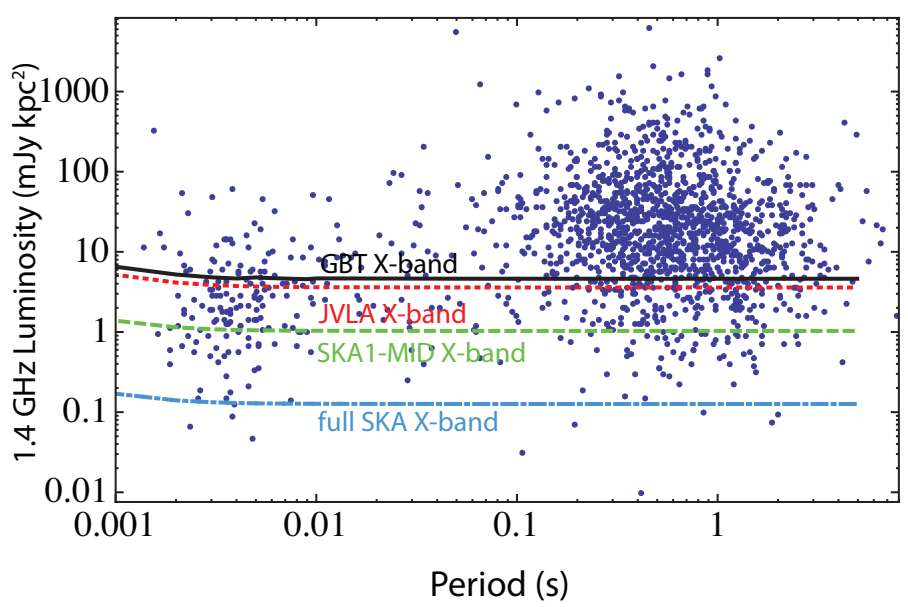

FIG. 4.- The $10 \sigma$ sensitivities of GBT, VLA, SKA1-MID and SKA 30-hour X-band integrations to the known Galactic pulsar population, if placed at the distance of the Galactic Center, assuming the weak-scattering case. As in Fig. 1 the dots show the $1.4 \mathrm{GHz}$ luminosity of the known pulsar population (Manchester et al. 2005) plotted versus pulsar period, while the solid, dashed, dotted and dash-dotted curves show the $10 \sigma$ sensitivities for the GBT, VLA, SKA1-MID and full SKA, respectively. It is clear that deep X-band observations with existing telescopes (the GBT and the VLA) would be sensitive to a significant fraction ( $\gtrsim 30 \%$ ) of the known MSP population (as well as to $\gtrsim 65 \%$ of the entire known pulsar population), if located at the GC distance. ever, the strong-scattering scenario would require the full SKA to detect and time MSPs at the distance of the Galactic Center.
Parts of this research were conducted by the Australian Research Council Centre of Excellence for Allsky Astrophysics (CAASTRO), through project number CE110001020. NK acknowledges support from the Department of Science and Technology via a Swarnajayanti Fellowship, and also thanks ICRAR for support during a visit during which part of this work was carried out. JPM thanks Yuri Levin for engaging discussions relating to the topic of this work. We also thank an anonymous referee for suggestions that improved the clarity of this paper.

\section{REFERENCES}

Alpar, M. A., Cheng, A. F., Ruderman, M. A., \& Shaham, J. 1982, Nature, 300, 728

Bagchi, M., Lorimer, D. R., \& Chennamangalam, J. 2011, MNRAS, 418, 477

Bates, S. D., Johnston, S., Lorimer, D. R., Kramer, M., Possenti, A., Burgay, M., Stappers, B., Keith, M. J., Lyne, A., Bailes, M., McLaughlin, M. A., O'Brien, J. T., \& Hobbs, G. 2011, MNRAS, 411, 1575

Bates, S. D., Lorimer, D. R., \& Verbiest, J. P. W. 2013, MNRAS, 431, 1352

Bower, G. C., Deller, A., Demorest, P., Brunthaler, A., Eatough, R., Falcke, H., Kramer, M., Lee, K. J., \& Spitler, L. 2014, ApJL, 780, L2

Camilo, F., Lorimer, D. R., Freire, P., Lyne, A. G., \& Manchester, R. N. 2000, ApJ, 535, 975

Campana, S., Colpi, M., Mereghetti, S., Stella, L., \& Tavani, M. 1998, A\&ARv, 8, 279

Chennamangalam, J. \& Lorimer, D. R. 2014, MNRAS, 440, L86

Cordes, J. M. \& Lazio, T. J. W. 1997, ApJ, 475, 557

Deneva, J. S. 2010, Ph.D. thesis, Cornell University

Deneva, J. S., Cordes, J. M., \& Lazio, T. J. W. 2009, ApJL, 702, L177

Dewdney, P. E., Turner, W., Millenaar, R., McCool, R., Lazio, J., \& Cornwell, T. J. 2013, SKA Report SKA-TEL-SKO-DD-001

Dexter, J. \& O'Leary, R. M. 2014, ApJL, 783, L7

Eatough, R. P., Falcke, H., Karuppusamy, R., Lee, K. J., Champion, D. J., Keane, E. F., Desvignes, G., Schnitzeler, D. H. F. M., Spitler, L. G., Kramer, M., Klein, B., Bassa, C., Bower, G. C., Brunthaler, A., Cognard, I., Deller, A. T., Demorest, P. B., Freire, P. C. C., Kraus, A., Lyne, A. G., Noutsos, A., Stappers, B., \& Wex, N. 2013, Nature, 501, 391

Faucher-Giguère, C.-A. \& Loeb, A. 2011, MNRAS, 415, 3951

Frail, D. A., Diamond, P. J., Cordes, J. M., \& van Langevelde, H. J. 1994, ApJL, 427, L43

Genzel, R., Thatte, N., Krabbe, A., Kroker, H., \& Tacconi-Garman, L. E. 1996, ApJ, 472, 153

Johnston, S., Kramer, M., Lorimer, D., Lyne, A., McLaughlin, M., Klein, B., \& Manchester, R. 2006, MNRAS, 373, L6

Johnston, S., Walker, M. A., van Kerkwijk, M. H., Lyne, A. G., \& D'Amico, N. 1995, MNRAS, 274, L43
Jouteux, S., Ramachandran, R., Stappers, B. W., Jonker, P. G., $\&$ van der Klis, M. 2002, A\&A, 384, 532

Keane, E. F. \& Kramer, M. 2008, MNRAS, 391, 2009

Kennea, J. A., Burrows, D. N., Kouveliotou, C., Palmer, D. M., Göğüs, E., Kaneko, Y., Evans, P. A., Degenaar, N., Reynolds, M. T., Miller, J. M., Wijnands, R., Mori, K., \& Gehrels, N. 2013, ApJL, 770, L24

Knispel, B. 2011, PhD thesis, Max-Planck-Institut für Gravitationsphysik, Hannover, Germany

Knispel, B., Eatough, R. P., Kim, H., Keane, E. F., Allen, B., Anderson, D., Aulbert, C., Bock, O., Crawford, F., Eggenstein, H.-B., Fehrmann, H., Hammer, D., Kramer, M., Lyne, A. G., Machenschalk, B., Miller, R. B., Papa, M. A., Rastawicki, D., Sarkissian, J., Siemens, X., \& Stappers, B. W. 2013, ApJ, 774, 93

Kramer, M., Klein, B., Lorimer, D., Müller, P., Jessner, A., \& Wielebinski, R. 2000, in Astronomical Society of the Pacific Conference Series, Vol. 202, IAU Colloq. 177: Pulsar

Astronomy - 2000 and Beyond, ed. M. Kramer, N. Wex, \& R. Wielebinski, 37

Kramer, M., Xilouris, K. M., Lorimer, D. R., Doroshenko, O., Jessner, A., Wielebinski, R., Wolszczan, A., \& Camilo, F. 1998, ApJ, 501, 270

Lazio, T. J. W. \& Cordes, J. M. 1998, ApJ, 505, 715

Liu, K., Wex, N., Kramer, M., Cordes, J. M., \& Lazio, T. J. W. 2012, ApJ, 747, 1

Lo, K. Y., Backer, D. C., Ekers, R. D., Kellermann, K. I., Reid, M., \& Moran, J. M. 1985, Nature, 315, 124

Lorimer, D. R., Faulkner, A. J., Lyne, A. G., Manchester, R. N., Kramer, M., McLaughlin, M. A., Hobbs, G., Possenti, A., Stairs, I. H., Camilo, F., Burgay, M., D'Amico, N., Corongiu, A., \& Crawford, F. 2006, MNRAS, 372, 777

Macquart, J., Kanekar, N., Frail, D. A., \& Ransom, S. M. 2010, ApJ, 715, 939

Manchester, R. N., Hobbs, G. B., Teoh, A., \& Hobbs, M. 2005 , AJ, 129, 1993

Maron, O., Kijak, J., Kramer, M., \& Wielebinski, R. 2000, A\&AS, 147, 195 
Mori, K., Gotthelf, E. V., Zhang, S., An, H., Baganoff, F. K., Barrière, N. M., Beloborodov, A. M., Boggs, S. E., Christensen, F. E., Craig, W. W., Dufour, F., Grefenstette, B. W., Hailey, C. J., Harrison, F. A., Hong, J., Kaspi, V. M., Kennea, J. A., Madsen, K. K., Markwardt, C. B., Nynka, M., Stern, D., Tomsick, J. A., \& Zhang, W. W. 2013, ApJL, 770, L23

Muno, M. P., Lu, J. R., Baganoff, F. K., Brandt, W. N., Garmire, G. P., Ghez, A. M., Hornstein, S. D., \& Morris, M. R. 2005, ApJ, 633, 228

Olausen, S. A. \& Kaspi, V. M. 2014, ApJS, 212, 6

Pfahl, E. \& Loeb, A. 2004, ApJ, 615, 253

Ransom, S. M., Cordes, J. M., \& Eikenberry, S. S. 2003, ApJ, 589,911

Ransom, S. M., Eikenberry, S. S., \& Middleditch, J. 2002, AJ, 124, 1788

Ransom, S. M., Hessels, J. W. T., Stairs, I. H., Freire, P. C. C., Camilo, F., Kaspi, V. M., \& Kaplan, D. L. 2005, Science, 307, 892

Reich, W., Reich, P., \& Fuerst, E. 1990, A\&AS, 83, 539

Schödel, R., Eckart, A., Alexander, T., Merritt, D., Genzel, R., Sternberg, A., Meyer, L., Kul, F., Moultaka, J., Ott, T., \& Straubmeier, C. 2007, A\&A, 469, 125

Shannon, R. M. \& Johnston, S. 2013, MNRAS, 435, L29
Siemion, A., Bailes, M., Bower, G., Chennamangalam, J., Cordes, J., Demorest, P., Deneva, J., Desvignes, G., Ford, J., Frail, D., Jones, G., Kramer, M., Lazio, J., Lorimer, D., McLaughlin, M., Ransom, S., Roshi, A., Wagner, M., Werthimer, D., \&

Wharton, R. 2013, in IAU Symposium, Vol. 291, IAU Symposium, ed. J. van Leeuwen, 57

Spitler, L. G., Lee, K. J., Eatough, R. P., Kramer, M., Karuppusamy, R., Bassa, C. G., Cognard, I., Desvignes, G., Lyne, A. G., Stappers, B. W., Bower, G. C., Cordes, J. M., Champion, D. J., \& Falcke, H. 2014, ApJL, 780, L3

Verbunt, F. 1987, ApJ, 312, L23

Verbunt, F. \& Hut, P. 1987, in IAU Symposium, Vol. 125, The Origin and Evolution of Neutron Stars, ed. D. J. Helfand \& J.-H. Huang, 187

Wharton, R. S., Chatterjee, S., Cordes, J. M., Deneva, J. S., \& Lazio, T. J. W. 2012, ApJ, 753, 108

Wood, K. S., Norris, J. P., Hertz, P., Vaughan, B. A., Michelson, P. F., Mitsuda, K., Lewin, W. H. G., van Paradijs, J., Penninx, W., \& van der Klis, M. 1991, ApJ, 379, 295

Zhang, F., Lu, Y., \& Yu, Q. 2014, ApJ, 784, 106 